\title{
Electroacupuncture is Beneficial for Primary Dysmenorrhea: The Evidence from Meta-Analysis of Randomized Controlled Trials
}

\author{
Si-yi Yu, ${ }^{1}$ Zheng-tao $\mathrm{Lv},{ }^{2}$ Qing Zhang, ${ }^{1}$ Sha Yang, ${ }^{1}$ Xi Wu, ${ }^{1}$ You-ping Hu, ${ }^{1}$ Fang Zeng, \\ Fan-rong Liang, ${ }^{1}$ and Jie Yang ${ }^{1}$ \\ ${ }^{1}$ The 3rd Teaching Hospital, Chengdu University of Traditional Chinese Medicine, Chengdu 610075, China \\ ${ }^{2}$ Department of Orthopedics, Tongji Hospital, Tongji Medical College, Huazhong University of Science and Technology, Wuhan, China
}

Correspondence should be addressed to Fan-rong Liang; acuresearch@126.com and Jie Yang; jenny_yang_jie@126.com

Received 30 July 2017; Revised 15 October 2017; Accepted 2 November 2017; Published 5 December 2017

Academic Editor: Thomas Lundeberg

Copyright @ $2017 \mathrm{Si}$-yi Yu et al. This is an open access article distributed under the Creative Commons Attribution License, which permits unrestricted use, distribution, and reproduction in any medium, provided the original work is properly cited.

\begin{abstract}
Electroacupuncture (EA) is considered to be a promising alternative therapy to relieve the menstrual pain for primary dysmenorrhea (PD), but the conclusion is controversial. Here, we conducted a systematic review and meta-analysis specifically to evaluate the clinical efficacy from randomized controlled trials (RCTs) on the use of EA in patients with PD. PubMed, Embase, ISI Web of Science, CENTRAL, CNKI, and Wanfang were searched to identify RCTs that evaluated the effectiveness of EA for PD. The outcome measurements included visual analogue scale (VAS), verbal rating scale (VRS), COX retrospective symptom scale (RSS), and the curative rate. Nine RCTs with high risk of bias were included for meta-analysis. The combined VAS 30 minutes after the completion of intervention favoured EA at SP6 when compared with EA at GB39, nonacupoints, and waiting-list groups. EA was superior to pharmacological treatment when the treatment duration lasted for three menstrual cycles, evidenced by significantly higher curative rate. No statistically significant differences between EA at SP6 and control groups were found regarding the VRS, RSS-COX1, and RSS-COX2. The findings of our study suggested that EA can provide considerable immediate analgesia effect for PD. Additional studies with rigorous design and larger sample sizes are needed.
\end{abstract}

\section{Introduction}

Primary dysmenorrhea (PD), or menstrual pain without discernable organic causes, is the most prevalent gynecologic complaint in young women. The prevalence of primary dysmenorrhea ranges from $20 \%$ to $90 \%$ of menstruating women [1], with severe pain perceived in $10 \%$ to $20 \%$ of the studied female adolescents [2]. The painful menstrual cramps experienced by women with PD can be considerably disabling, having been likened to renal colic pain [3]. Previous studies have proposed that PD is associated with prostaglandins (PGs), which may play a vital role in modulating hyperalgesia and inflammatory pain and causing uterine contraction [4]. Although PD is not life-threatening, the degree of pain severely affects women's quality of life and ability to study or work normally $[5,6]$. Therefore, an improved understanding and treatment for PD are urgent and compulsory.
Nowadays, the treatment for PD includes a variety of pharmacological and nonpharmacological methods. On account of the PG-based etiology of PD, the current frontline pharmacological treatment for PD is nonsteroidal antiinflammatory drugs (NSAIDs) [7]. Undeniably, NSAIDs have well-documented efficacy against menstrual pain; however, they have a high failure rate of $20 \%-30 \%$ [8]. Moreover, painkillers are also associated with an increased risk of adverse events (AEs), including the digestive tract, liver, and kidney [9]. Accordingly, the search for a low-risk and effective nonpharmacological therapy to relieve menstrual pain represents urgent clinical demand.

Acupuncture, an integral part of Traditional Chinese Medicine (TCM) [10], has been verified to be effective in relieving the menstrual pain and reducing the symptoms of primary dysmenorrhea through plenty of randomized controlled trials (RCTs) [11-13] and systematic reviews [14, 15]. 
As one of the oldest nonpharmacological therapies, manual acupuncture (MA) mediates its therapeutic effect through the insertion of needles into specific points in the body called acupoints [16]. In the past decades, electroacupuncture (EA), as a means combining traditional acupuncture with modern electrotherapy, has been widely used in China and elsewhere around the world due to its accurate, quantifiable, and repeatable parameters of intensity, frequency, and duration $[17,18]$. So far, the mechanism of EA for PD has not been fully illuminated, but some experimental investigations indicated that the EA could reduce the expression levels of prostaglandin (PGF2 $\alpha$ ) [19], the IP3 [20], and the peripheral blood T lymphocyte subsets [21] in a rat model of PD. To date, although many RCTs have demonstrated significant efficacy and safety of EA for PD [22], further investigations are required to draw a reliable conclusion about the effectiveness of this treatment.

However, no published systematic review focused specifically on the effect of EA for PD. Although the results of several published systematic reviews with meta-analysis [14, 15, 23-27] of acupuncture for PD suggested a positive effect of EA, their results could not be confirmed partially due to the small number and methodological flaws of the included studies [22, 28-30]. Moreover, both manual acupuncture and electrical acupuncture are typically lumped together to constitute scientific evidence on "acupuncture" among all of the above systematic reviews. Significantly, Langevin et al. recently reported that EA and MA treatments are not interchangeable and thus must be separately identified for accurate study [31]. Further, Zhang et al. summarized the mechanisms of MA-EA on persistent pain, indicating that EA displays greater analgesic effects for different types of pain in comparison to MA [32]. Therefore, with the publication of a fair number of studies on EA for PD in recent years, we conducted this systematic review to critically evaluate the current evidence from RCTs on the use of EA in patients with PD.

\section{Methods}

This systematic review was performed according to the Preferred Reporting Items for Systematic Reviews and MetaAnalyses (PRISMA) guidelines.

2.1. Literature Search Strategy. Six electronic databases, PubMed, Embase, ISI Web of Science, CENTRAL, CNKI, and Wanfang, were searched to identify potentially eligible studies. All the above databases were searched from inception up to the latest issue (April 2017) without language restriction. Free text terms and Medical Subject Headings (MeSH) were combined for literature retrieval; $\mathrm{MeSH}$ terms were modified according to the specification of each database. The literature search strategy was as follows: ("Electroacupuncture" [Mesh] or electroacupuncture or electric acupuncture) and ("Dysmenorrhea" [Mesh] or dysmenorrhea or menstrual pain or painful menstruation). In CNKI and Wanfang, we searched "Dian zhen" and "Tong jing" for potential eligible Chinese publications. The bibliographies of relevant systematic reviews were also manually searched to ensure that all relevant studies could be included.

2.2. Types of Participants. To be included in our systematic review and meta-analysis, participants enrolled in included studies should be diagnosed as primary dysmenorrhea. Following conditions leading to secondary dysmenorrhea, such as endometriosis, uterine myoma, ovarian cyst, intrauterine synechia, or intrauterine devices, should be excluded from the study.

2.3. Types of Intervention. Patients in experimental groups were required to receive EA treatment; no specific restriction on current intensity was imposed. RCTs that combined EA and other therapeutic approaches to treat women with PD were excluded.

2.4. Types of Control. Patients with PD in control groups mainly received pharmacological treatments, EA at unrelated acupoints, or EA at nonacupoint locations; no specific type of analgesics was imposed. Patients in waiting-list groups were also included.

2.5. Outcome Measurements. The primary outcome measure was subjective pain measured by a standard $100 \mathrm{~mm}$ VAS 30 minutes after the completion of intervention. We selected verbal rating scale (VRS) and COX retrospective symptom scale (RSS) as the secondary outcome. The latter is a menstrual symptom measure with high reliability and sensitivity; it is comprised of two subscales, a total frequency rating score (RSS-COX1) and an average severity score (RSSCOX2). The 7-point VRS defines menstrual pain according to the limitation of ability to daily work, systematic symptoms, and need for additional analgesics. The curative rate was also included in our meta-analysis.

2.6. Types of Publication. Only RCTs regarding the efficacy of EA on PD were included in our study. Case control studies, cohort studies review articles, and animal experiments were excluded.

2.7. Data Extraction. Two investigators (Si-yi Yu and Zhengtao Lv) screened each article independently and each was blinded to the findings of the other reviewer. According to the predetermined inclusion criteria, two reviewers performed strict screening to identify qualified articles independently, and they extracted data from these eligible articles using a standardized data collection form, which included first author, year of the publication, sample size in each group, details of EA intervention and control treatment, main outcome assessment, and duration of treatment.

Any disagreement between the two reviewers was resolved through discussion until a consensus was reached. The third review author (Fan-rong Liang) was consulted if a consensus could not be reached.

2.8. Risk of Bias Assessment. The Cochrane Collaboration's tool was employed for the assessment of risk of bias in 
selected RCTs, which was based on seven items: random sequence generation, allocation concealment, blinding of participants and personnel, blinding of outcome assessment, incomplete outcome data, selective reporting, and other sources of bias. Two reviewers assessed the risk of bias among studies independently; the results were compared afterwards. Disagreements regarding the risk of bias assessment were settled by discussion and consensus between reviewers.

2.9. Statistical Analysis. The enrolled participants were dichotomized into cured and not cured to express the intervention effect; risk ratio (RR) and the associated 95\% confidence intervals (CIs) were calculated for clinical effect. For continuous variables, mean difference (MD) for changes from baseline was calculated using the same methodology. A $P$ value less than 0.1 from the $Q$ statistic was considered as statistically significant heterogeneity. $I^{2}$ values of $0 \%, 25 \%$, $50 \%$, and $75 \%$ corresponded to no, low, moderate, and high levels of heterogeneity, respectively [33]. The consistency of results across studies was assessed by $\tau^{2}$ statistic for relevant heterogeneity [34] and $I^{2}$ statistic for statistical heterogeneity [33]. We pooled the estimates using the fixedeffects model when no significant heterogeneity was detected [35]. Otherwise, a random-effects model was used [36].

Subgroup analysis by control intervention was conducted. Sensitivity analysis was conducted by removing studies with relatively small sample sizes (less than 20 subjects in EA group or control group) to determine whether small sample sizes contributed to the between-study heterogeneity. Forest plots and funnel plots were generated via RevMan 5.3 (Copenhagen: the Nordic Cochrane Centre, the Cochrane Collaboration, 2014).

\section{Results}

3.1. Literature Search. The systematic literature search yielded a total of 282 potentially relevant articles: 28 from PubMed, 30 from Embase, 62 from ISI Web of Science, 16 from CENTRAL, 78 from CNKI, and 68 from Wanfang. After the removal of duplicates, 156 studies entered the stage of title and abstract screening and 11 of them were downloaded for the full-text screening. Two studies were subsequently removed because one was non-RCT and the other used unsuitable comparison. Additionally, we searched seven systematic reviews related to acupuncture therapy and PD; their included studies that used EA to treat PD were also selected by our current systematic review. Finally, nine studies [28, 30,37-43] were deemed eligible for inclusion in the meta-analysis. The flow chart of literature search was presented in Figure 1.

3.2. Main Characteristics of Included Studies. All the selected RCTs were carried out in mainland China and published from 2007 to 2016 . Women with a history of regular menstruation who were diagnosed as PD were enrolled in our included studies. Among these RCTs, SP6 was the most commonly selected acupoint for EA administration. The duration of EA treatment was noted to vary; three two-arm parallel studies $[39,40,43]$ compared EA with pharmacological therapy for

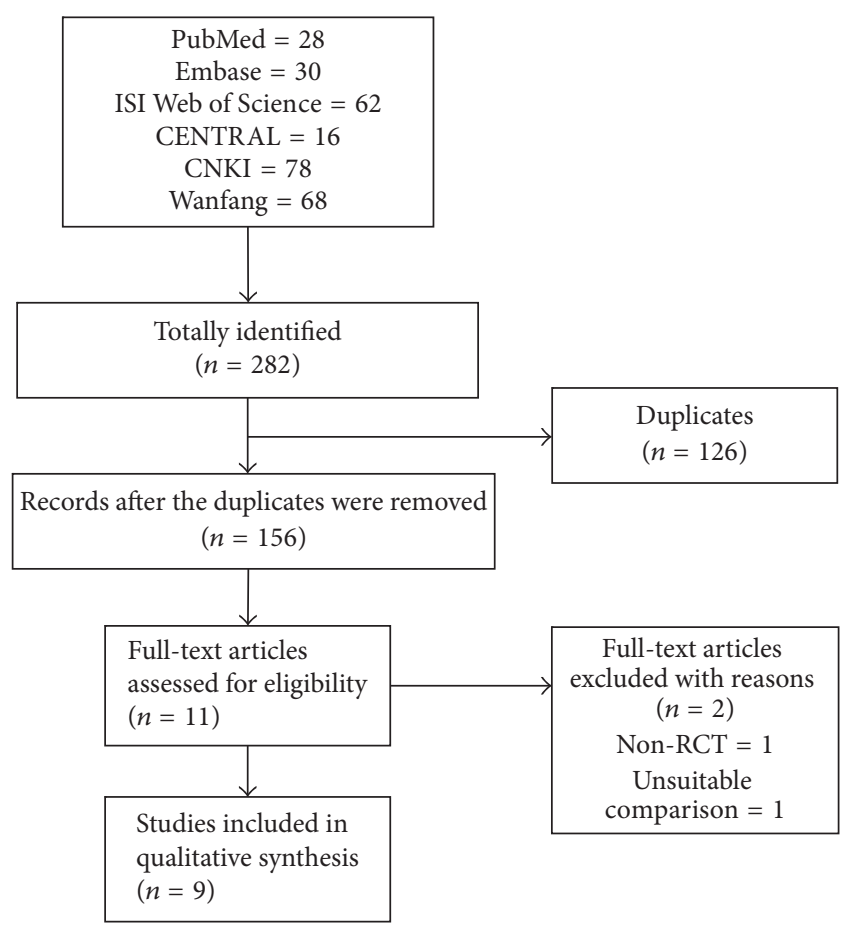

FIGURE 1: Flow diagram of literature selection.

three menstrual cycles. In these aforementioned studies, EA interventions were introduced from the first day of the onset of menstruation and continued for another four days. After three months' treatment, the clinical effect of EA treatment was evaluated using a dichotomous scale (cured and not cured). The remaining six studies [28, 30, 37, 38, 41, 42] were performed based on three-arm or four-arm parallel design; pain intensity was assessed 30 minutes after the completion of EA administration. The main characteristics of included RCTs were summarized in Table 1.

3.3. Methodological Quality. Cochrane's Handbook was utilized for the assessment of methodological quality. All the included studies reported the suggested randomization, but two $[40,42]$ studies failed to provide the method of random sequence generation. Regarding the allocation concealment, three studies [28, 37, 41] reported the details of allocation concealment procedure. When it comes to blinding of personnel and participants, all trials were judged to have a high risk of bias because it was unfeasible to blind the acupuncturists who administered EA. In terms of blinding of outcome assessment, five studies [28, 30, 37, 38, 41] were judged to have a low risk of bias. The reviewers' judgements about each risk of bias item were presented in Figure 2 .

3.4. VAS. In order to determine the immediate analgesic effect of EA on PD, six studies [28, 30, 37, 38, 41, 42] evaluated menstrual pain 30 minutes after the end of first EA administration using the $100 \mathrm{~mm}$ VAS. Significant heterogeneity among included studies was observed; thus the random-effects model was used. The pooled results showed that EA at SP6 was better in pain relief compared to EA at 


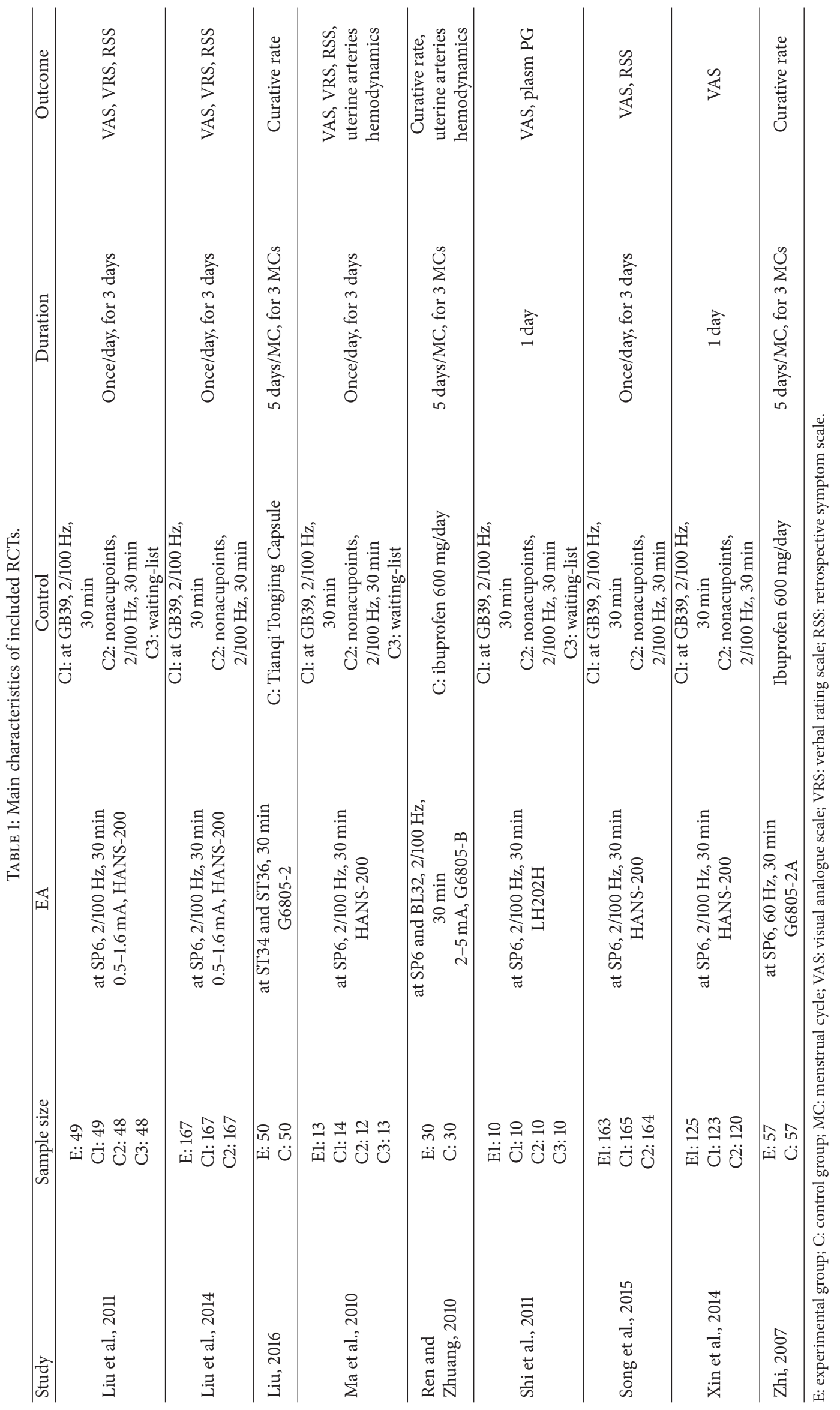




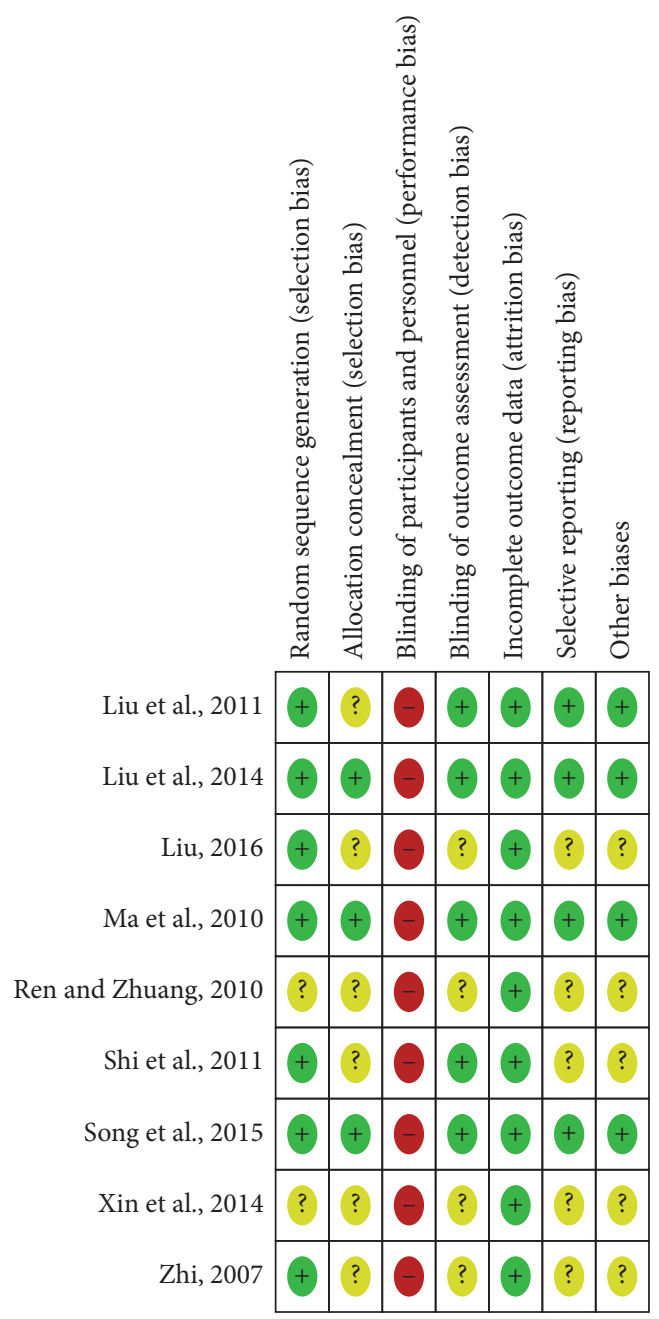

FIGURE 2: Risk of bias summary: reviewing authors' judgements about each risk of bias item for each included study.

GB39 $\left(\tau^{2}=127.47, \chi^{2}=79.71, \mathrm{df}=5\right.$, and $I^{2}=94 \%$; MD: 11.27 ; 95\% CI: 1.76, 20.78), EA at nonacupoints $\left(\tau^{2}=65.96, \chi^{2}=\right.$ 46.80, $\mathrm{df}=5$, and $I^{2}=89 \%$; MD: 9.33; 95\% CI: 2.18, 16.47), and waiting-list $\left(\tau^{2}=117.51, \chi^{2}=12.43, \mathrm{df}=2\right.$, and $I^{2}=84 \%$; MD: 27.15; 95\% CI: 13.74, 40.55) groups (Figure 3).

3.5. VRS. The 7-point VRS was employed by three studies $[28,30,37]$ to assess menstrual pain in the intervention menstrual cycle based on loss of efficiency of work, systematic symptoms, and need for additional analgesics. Compared with GB39 $\left(\chi^{2}=3.13, \mathrm{df}=2\right.$, and $I^{2}=36 \%$; MD: 0.16 ; 95\% CI: $-0.04,0.37)$, nonacupoints $\left(\chi^{2}=0.48, \mathrm{df}=2\right.$, and $I^{2}=0 \%$; MD: 0.05 ; 95\% CI: $-0.16,0.25)$, and waiting-list $\left(\chi^{2}=1.62\right.$, $\mathrm{df}=1$, and $I^{2}=38 \%$; MD: 0.25 ; 95\% CI: $\left.-0.14,0.63\right)$ groups, EA at SP6 showed no better effect in improving VRS score (Figure 4).

3.6. RSS-COX1 and RSS-COX2. RSS-COX1 refers to the total frequency of menstrual pain conditions that patients experienced in the intervention menstrual cycle, with lower score indicating better health. Obvious between-study heterogeneity was detected, so the random-effects model was used. The combined results from three studies [28, 30, 37] suggested that EA at SP6 had equivalent effect to EA at GB39 $\left(\tau^{2}=4.77, \chi^{2}=6.23, \mathrm{df}=2\right.$, and $I^{2}=68 \%$; MD: $0.41 ; 95 \% \mathrm{CI}$ : $-2.65,3.47)$, EA at nonacupoints $\left(\tau^{2}=3.66, \chi^{2}=5.09\right.$, df $=$ 2 , and $I^{2}=61 \%$; MD: 2.00 ; 95\% CI: $\left.-0.81,4.81\right)$, and waitinglist $\left(\tau^{2}=10.63, \chi^{2}=3.36, \mathrm{df}=1\right.$, and $I^{2}=70 \%$; MD: 1.45 ; $95 \%$ CI: $-3.86,6.76)$ groups in reducing the total frequency of menstrual pain conditions (Figure 5).

Four studies [28, 30, 37, 41] used RSS-COX2 to evaluate the average severity of menstrual pain in the intervention menstrual cycle. Since no significant heterogeneity across studies was observed, the fixed-effects model was employed for statistical analysis. The combined results indicated that EA at SP6 had similar effect in improving RSS-COX2 scores compared with EA at GB39 $\left(\chi^{2}=3.64, \mathrm{df}=3\right.$, and $I^{2}=17 \%$; MD: 0.26 ; 95\% CI: $-0.30,0.83), \mathrm{EA}$ at nonacupoints $\left(\chi^{2}=\right.$ 3.84 , df $=2$, and $I^{2}=22 \%$; MD: $\left.-0.10 ; 95 \% \mathrm{CI}:-0.68,0.49\right)$, and waiting-list $\left(\chi^{2}=0.77, \mathrm{df}=1\right.$, and $I^{2}=0 \%$; MD: $1.47 ; 95 \%$ CI: $-0.10,3.03$ ) groups (Figure 6).

3.7. Curative Rate. Three RCTs [39, 40, 43] adopted curative rate as outcome assessment; all of these three studies 


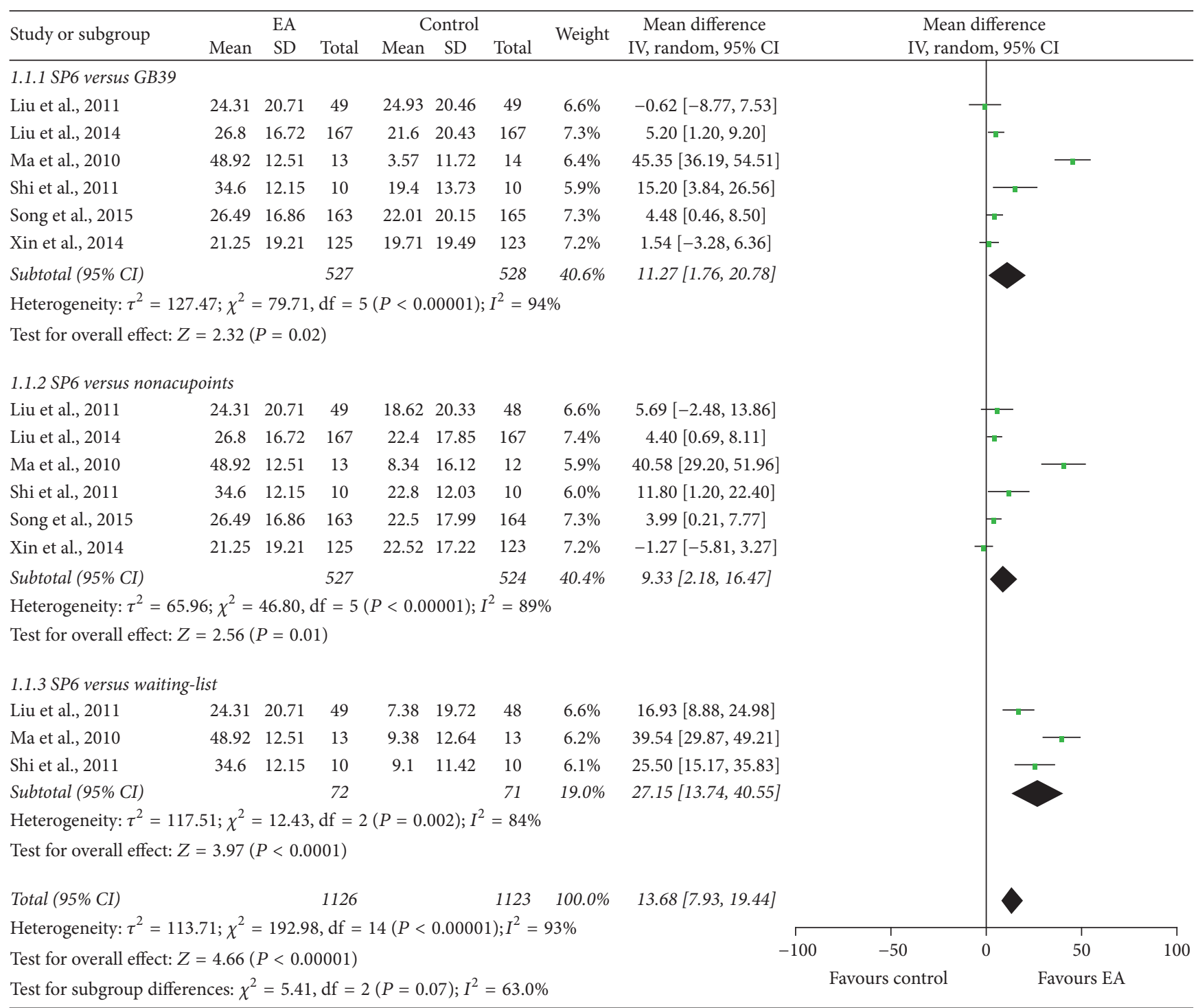

FIgURE 3: Forest plot of electroacupuncture versus control: VAS.

compared EA therapy with conventional drug therapy. In the studies of Ren and Zhuang and Zhi, patients in control groups received $600 \mathrm{mg}$ ibuprofen daily, while in Liu's study women received Tianqi Tongjing Capsules to alleviate menstrual pain. After three menstrual cycles' treatment, patients with PD were dichotomized as cured and not cured according to the "Standards for Diagnosis of Syndromes or Diseases of TCM and Evaluation of the Therapeutic Effect" [44]. "Cured" was defined as complete pain relief after three months' treatment without recurrence. "Not cured" referred to the situations where menstrual pain was not relieved and other related symptoms were not alleviated after three months' treatment. The study by Liu et al. was not included in the meta-analysis due to the uncertainty of the clinical effect of Tianqi Tongjing Capsule in PD. Liu and colleagues reported that EA at ST34 and ST36 was significantly better than Tianqi Tongjing Capsule in improving the curative rate (RR: 4.10; 95\% CI: 2.32, 9.25). Since no obvious between-study heterogeneity existed ( $P=0.16 ; I^{2}=49 \%$ ), the fixedeffects model was used for meta-analysis. The combination of curative rate suggested that EA was superior to ibuprofen when the treatment duration lasted for three menstrual cycles $\left(\chi^{2}=1.97\right.$ and $\mathrm{df}=1$; RR: 3.17; 95\% CI: 2.04, 4.92; $\left.P<0.00001\right)$ (Figure 7).

3.8. Sensitivity Analysis. Sensitivity analysis was conducted by removing RCTs with relatively small sample size and reevaluating the resulting effect. In the forest plot of VAS score, after the removal of $\mathrm{Ma}$ et al. and Shi et al. studies, heterogeneity across studies significantly decreased, and the conclusion regarding the immediate analgesic effect of EA at SP6 did not change (Figure 8).

3.9. Publication Bias. The funnel plots of VAS, VRS, RSSCOX1, and RSS-COX2 were presented in Figures 9, 10, 11, 


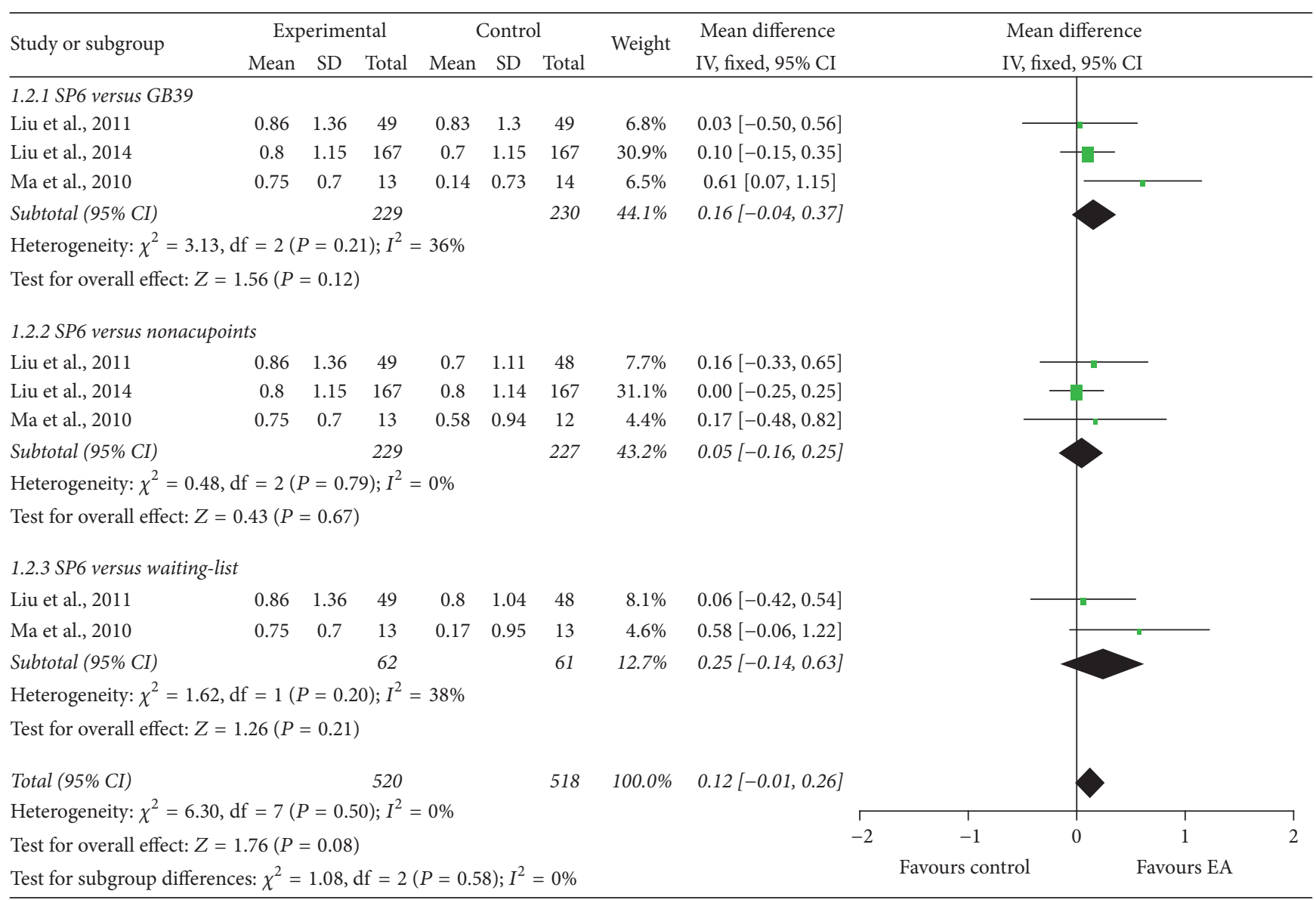

FIGURE 4: Forest plot of electroacupuncture versus control: VRS.

and 12. All the funnel plots presented no asymmetry, suggesting no obvious publication bias.

\section{Discussion}

4.1. Overview of Findings. A total of nine studies involving 1951 participants examining the effects of EA therapy on the management of PD were identified in this systematic review and meta-analysis. In our meta-analysis, the immediate and long-term therapeutic effects of EA were evaluated through an analysis of six and three pooled RCTs, respectively. In terms of pain intensity, six studies reported positive results using the VAS $[28,30,37,38,41,42]$, suggesting that EA at SP6 acupoint had a significant immediate effect on menstrual pain compared with treatment-irrelevant acupoint (GB39), nonacupoint, and waiting-list control. The goal of therapy is to minimize the pelvic pain that starts with the onset of the menstrual flow. Currently, our results suggest that EA stimulation at classic acupoint could alleviate the pain at once when compared with controls. The immediate analgesic effects of EA may be associated with the activation of the endogenous opioid system, which has been supported by plenty of experimental evidence [45-47].

Sanyinjiao (SP6), located medially four-finger wide above the ankle, has been the major acupoint to be used for treating
PD since ancient times according to the meridian theory of Chinese acupuncture. Further, it is used most frequently in treating $\mathrm{PD}$ according to our previous data mining analysis from literature [48]. In addition, recent RCTs have shown that SP6 stimulation could relieve the abdominal pain and improve the menstrual pain-related symptoms $[49,50]$. Thus, SP6 is claimed as a key acupoint for PD. Conversely, GB39 is an acupoint of gallbladder meridian located at the same level of SP6 on the extremity, and it is usually used to treat migraine, stiff neck back pain, shoulder pain, and so forth, but there are few reports on treatment for gynecologic indications. Thus, SP6 is claimed as a key acupoint for PD and the treatment-irrelevant acupoint GB39 (in the same spinal segments of SP6) is used as control points.

However, no significant differences were found in EA at SP6 versus above controls for lowing menstrual symptoms assessed by RSS-COX1 [28, 30,37] and RSS-COX2 $[28,30,37,41]$, which was also supported by the metaanalysis regarding the influence of menstrual pain on daily life assessed by VRS [28, 30,37]. This finding does not support the positive effects of acupuncture on PD in the majority of previously published studies [51]. A possible explanation for this nonsignificant finding is that RSS-COX1 and RSS-COX2 were used to evaluate the total frequency and average severity of dysmenorrhea symptoms monthly [44], 


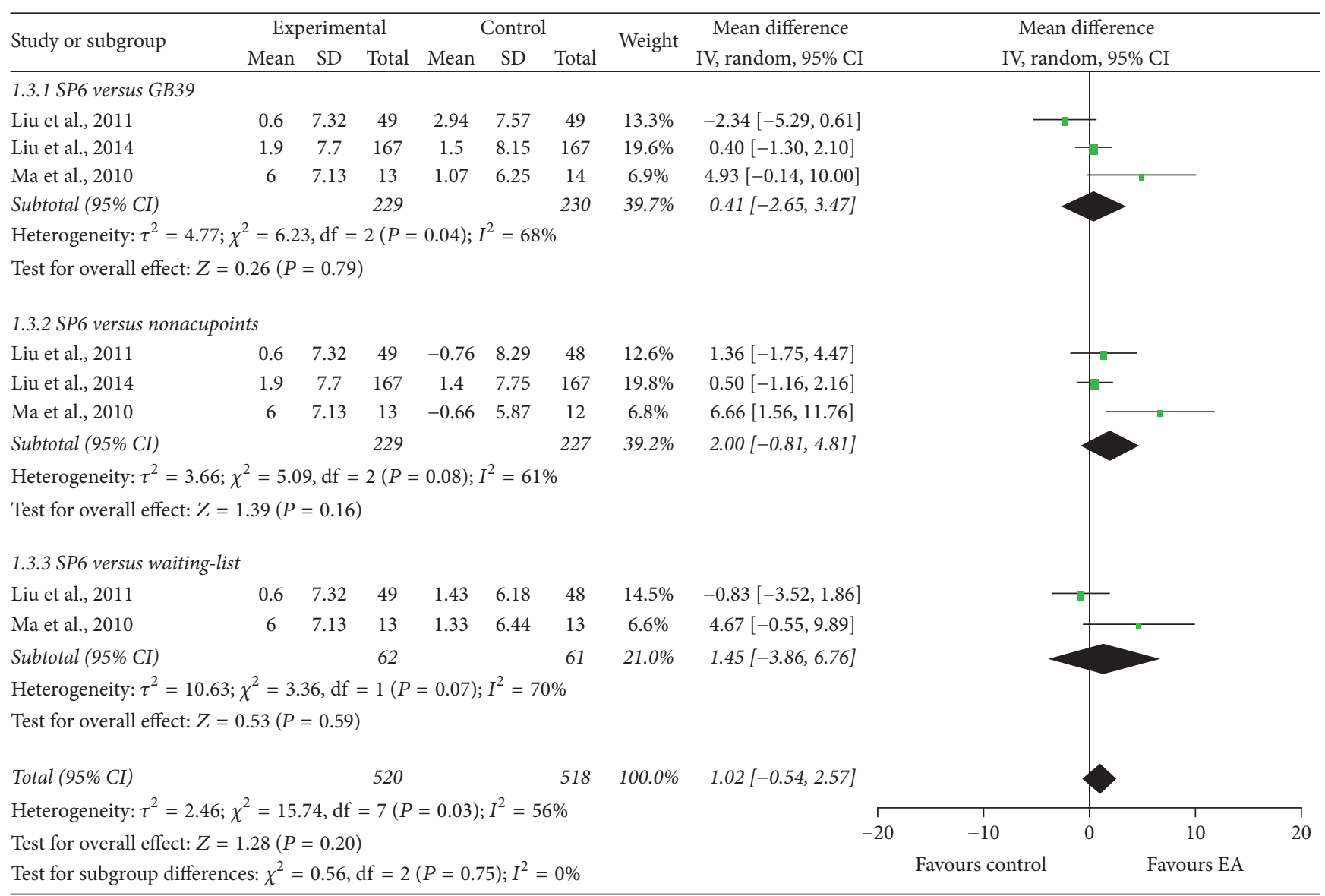

FIGURE 5: Forest plot of electroacupuncture versus control: RSS-COX1.

which should be assessed at end of the menstrual period and not immediately after interventions. Furthermore, immediate changes on menstrual pain after interventions assessed by VAS are more sensitive and understandable than VRS [52].

On the other hand, regarding curative rate, the outcome proven by three studies [39, 40, 43] showed that EA stimulation was more effective than conventional drug therapy after a course of treatment (three menstrual cycles or more). Notably, curative rate has not been validated and should be interpreted with caution. However, apart from curative rate, outcome measures of pain relief (VAS) and other menstrual symptoms (RSS-COX1 and RSS-COX2) have not been applied in the three studies to investigate the cumulative and long-term effect of EA on women with PD. Correspondingly, objective and quantitative assessments of PD should be collected by future RCTs to overcome the limitations of previous studies.

Consistent with our current report, some previous metaanalyses of nonpharmacological interventions on PD focused on the acupuncture [27], acupressure [25,53], moxibustion [54], aromatherapy massage [55], Chinese herbal medicine [56], transcutaneous electrical nerve stimulation [57], vitamin E [58], and oral Ginger [59]. Meanwhile, many metaanalyses about EA have emerged in the recent three years, indicating that EA could provide a positive therapeutic effect for cardiac anesthesia and intensive care [60], knee osteoarthritis [61], acute ischemic stroke [17], and tinnitus [62]. To the best of our knowledge, this is the first comprehensive systematic review and meta-analysis of RCTs on EA in the treatment of PD.

4.2. Limitations. The key strength of this study is that all the included RCTs were evaluated as "low" or "moderate" risk of bias in four domains based on the Cochrane collaboration RoB tool. There were also some limitations to consider in interpreting our study. First, our search did not include data in languages other than Chinese and English, which may generate a sampling bias. Further, although 4/9 trials were published in English, the populations involved in the included RCTs were all Chinese. No multicentered study with PD women of different races was gathered and thus EA therapy for non-Chinese populations still remains uncertain. Second, the methodological quality of the included trials was often suboptimal. Randomization, blinding, samplesize calculation, and the handling of all data should be reported specifically, as these are the principal standards of rigorous study design [63]. Although 7/9 studies described the specific methods of random sequence generation, only three studies declared allocation concealment. In addition, none of the included trials reported any details of blinding 


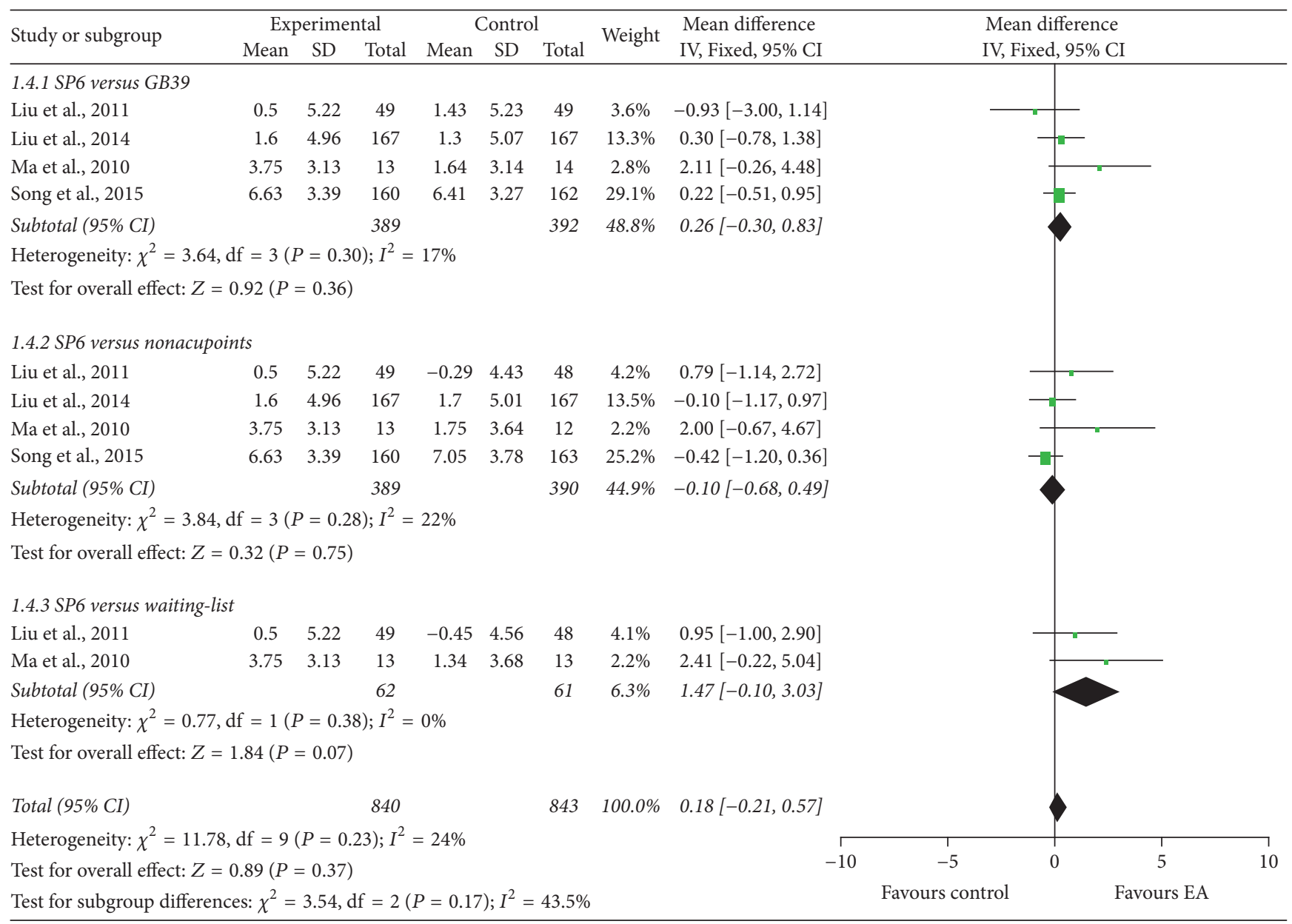

FIGURE 6: Forest plot of electroacupuncture versus control: RSS-COX2.

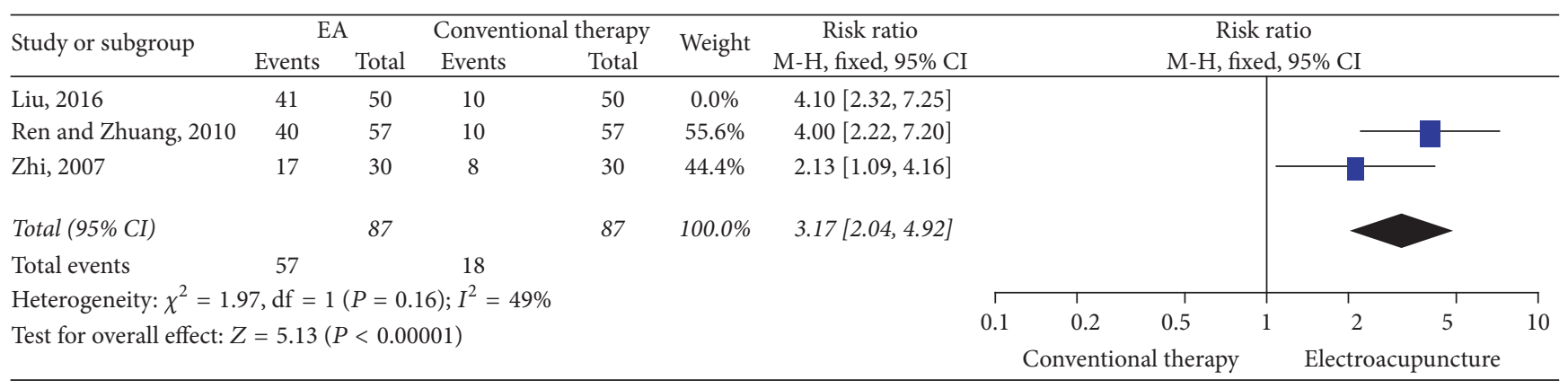

FIGURE 7: Forest plot of electroacupuncture versus control: curative rate.

or the sample-size estimation. Low quality of the included studies may cause overestimation of the treatment effects and thus limit our confidence in the results of this meta-analysis. Third, a certain degree of heterogeneity was observed in some of the meta-analyses in this systematic review. To gain a more in-depth understanding of the overall evidence of EA for PD, RCTs of different treatment schemes, time of application, duration of stimulation, and acupoints selected were included in our systematic review, which may give rise to clinical heterogeneity and thus may negatively affect our results. Finally, some RCTs did not use recognized reliability and validity outcome measurements on PD study, especially the clinically relevant outcomes (e.g., VAS, RSS-COX1, and RSS-COX2). Specifically, this review did not include data on the long-term efficacy of EA in reducing the abdominal pain or improving the menstrual symptoms, since none of the included RCTs evaluated these outcomes after a course of treatment. To judge whether EA is effective for treating PD in future studies, future trials on the evaluation of therapeutic effects should be in compliance with international standards. 


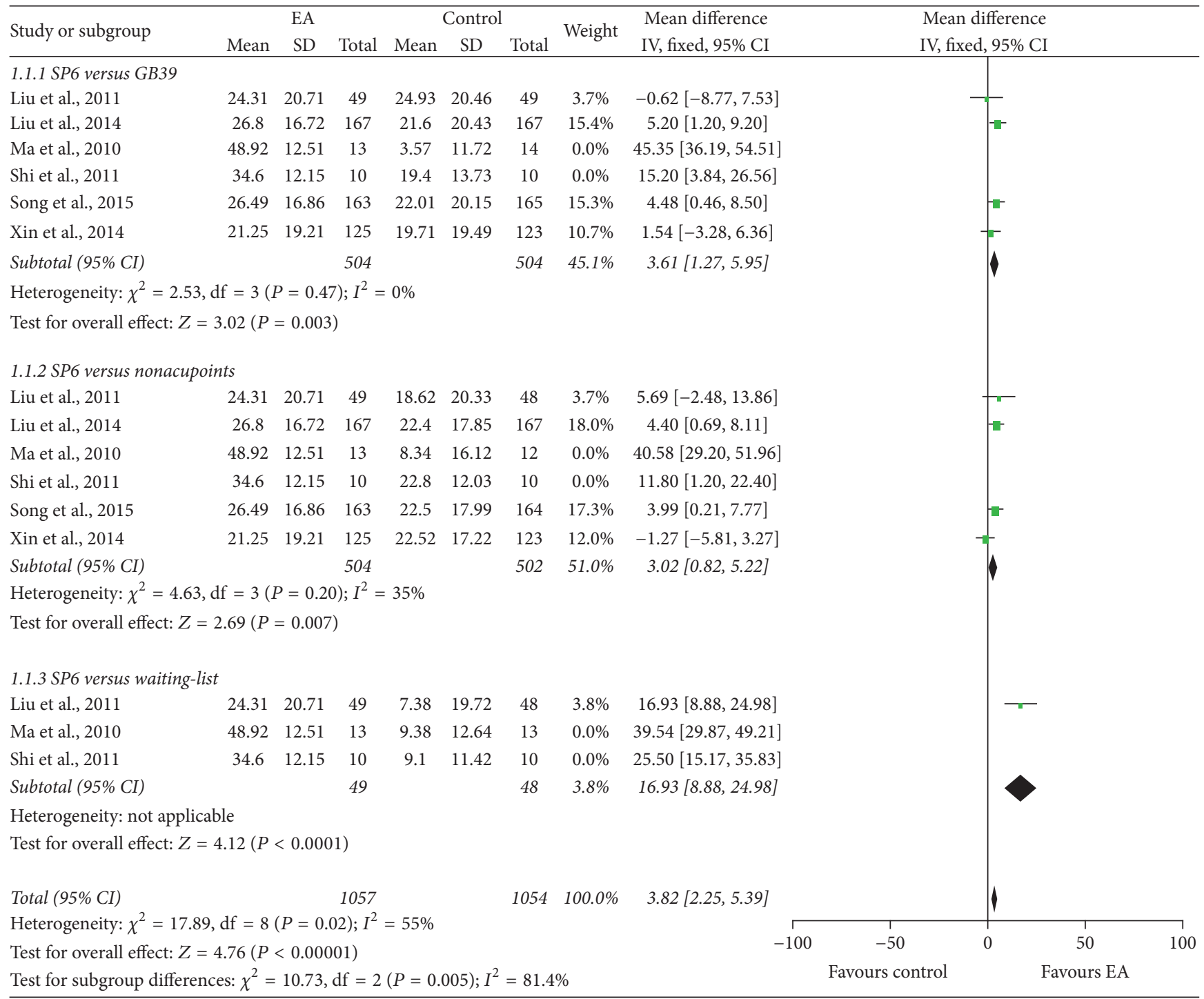

FIGURE 8: Sensitivity analysis by removing studies with relatively small sample sizes.

4.3. Implications for Practice. With the ever-growing interest in complementary and alternative treatments for chronic disease, there has increasingly been attention directed at EA for PD practices. In terms of pain intensity, six studies reported that EA at SP6 acupoint had a significant effect on cramping pain (assessed using a VAS) compared with GB39, nonacupoint, and waiting-list control in the short term. Specific acupoint is defined as points situated in meridian line with the strongest and the most concentrated power for certain disease. According to the principles of Chinese medicine, SP6 is the junction point of spleen, liver, and kidney meridians and is closely related to lower abdomen and uterus. Therefore, SP6 is commonly applied in clinical practice for alleviating dysmenorrhea, presenting preferred instant analgesic effect compared with irrelevant acupoint GB39 on gallbladder meridians and nonacupoints absent from meridian line. Moreover, there was greater prevalence of curative rate in the EA therapy compared with the pharmacological treatments in the long term. Taken together, our findings support that EA at SP6 acupoint should be recommended for patients with $\mathrm{PD}$.

4.4. Implications for Research. Considering the above limitations, more well-designed, rigorous, and large RCTs would facilitate an evidence base that can more decisively provide estimates of EA for PD. To improve methodological quality of clinical trials, further RCTs of EA for PD should use the CONSORT statement [60] and revised Standards for Reporting Interventions in Clinical Trials of Acupuncture (STRICTA) [61] as a guideline. Furthermore, clinically relevant outcomes, such as pain intensity and dysmenorrhearelated symptoms, should be addressed and evaluated using validated measurement scales such as a Short-Form McGill Pain Questionnaire or VAS for pain and the RSS-COX1 and RSS-COX2 for related symptoms. Finally, the electrical characteristics of EA, including the electric device, wave 


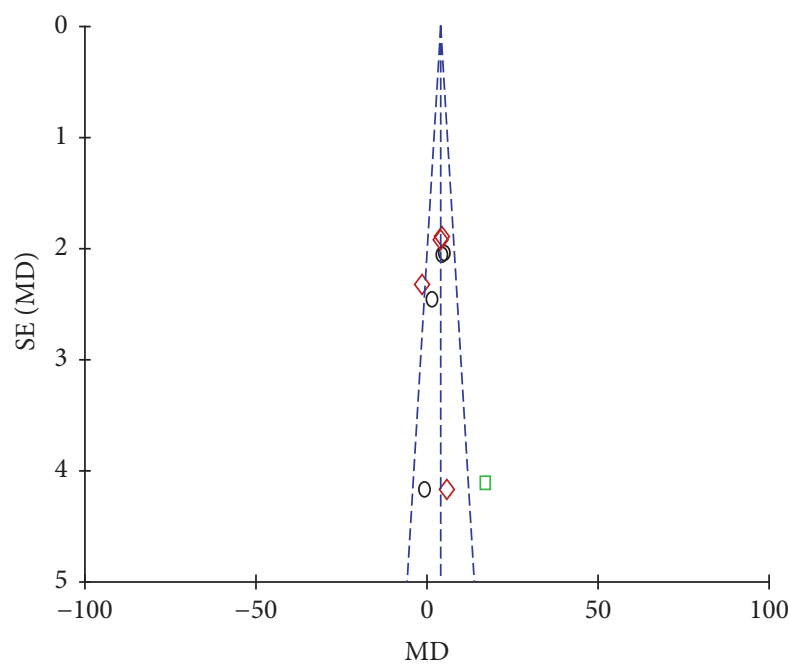

Subgroups

o SP6 versus GB39

$\diamond$ SP6 versus nonacupoints

$\checkmark$ SP6 versus waiting-list

FIGURE 9: Funnel plot of electroacupuncture versus control: VAS.

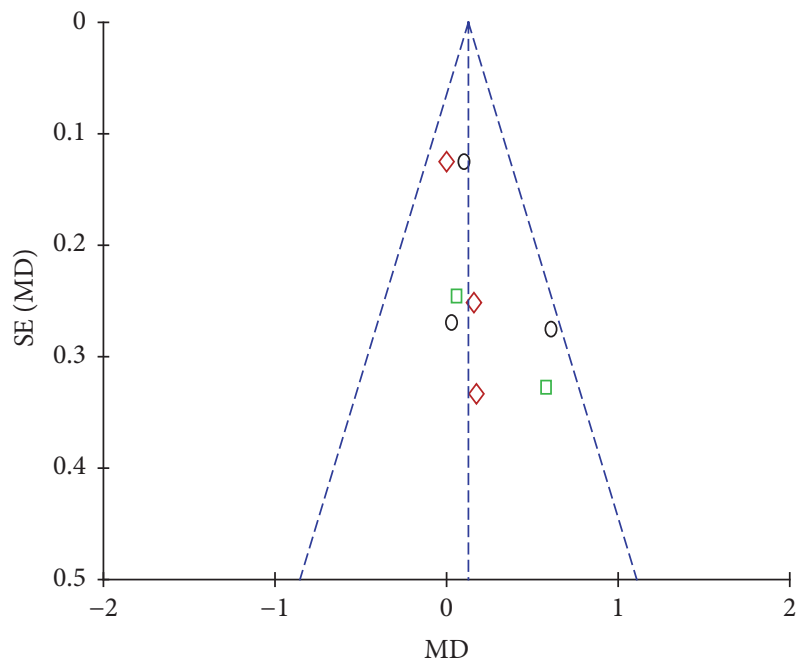

Subgroups

O SP6 versus GB39

$\diamond$ SP6 versus nonacupoints

૫ SP6 versus waiting-list

FIGURE 10: Funnel plot of electroacupuncture versus control: VRS.

length, and frequency, are worthy of further investigation in the future study.

\section{Conclusions}

Our findings indicated that EA at SP6 can provide considerable immediate analgesic effect for PD and its immediate effect of pain relieving seems to be superior to control interventions. Moreover, there was greater prevalence of curative rate in the EA treatment group compared with

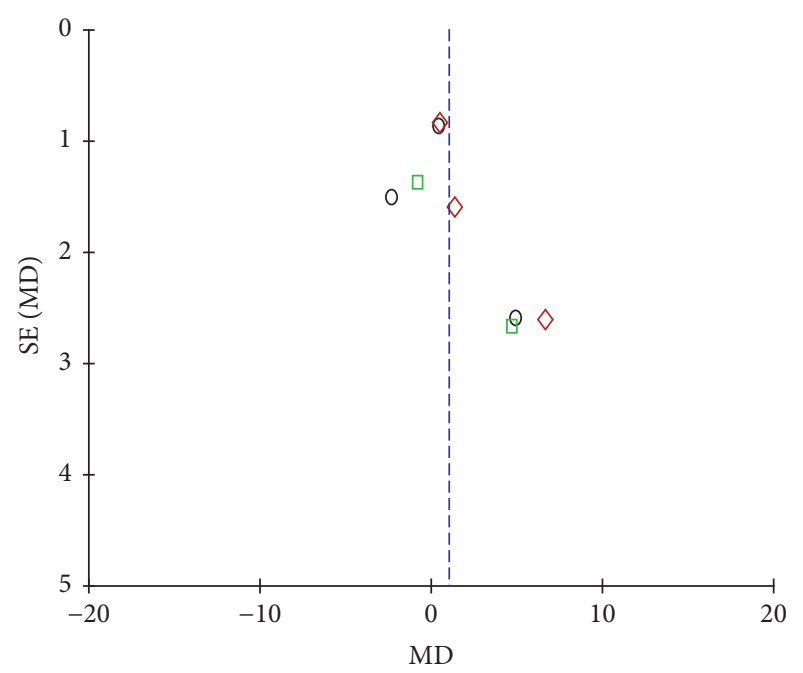
Subgroups
O SP6 versus GB39
$\diamond$ SP6 versus nonacupoints
$\square$ SP6 versus waiting-list

FIGURE 11: Funnel plot of electroacupuncture versus control: RSSCOX1.

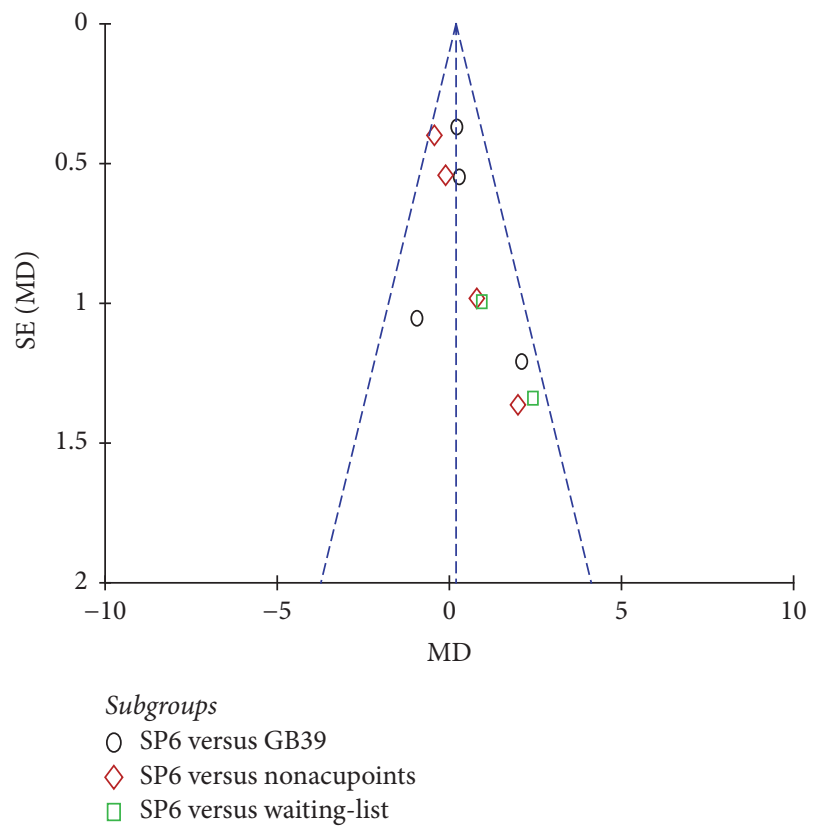

FIGURE 12: Funnel plot of electroacupuncture versus control: RSSCOX2.

the pharmacological treatments after a course of treatment. These results appear to be encouraging, but it should be considered at the same time that they are based on relatively low number of trials and relatively poor methodological quality of the primary studies. Hence, future research should be designed strictly and comprehensively to provide unbiased evidence about the efficacy of EA in the treatment of PD. 


\section{Conflicts of Interest}

The authors declare that they have no conflicts of interest.

\section{Authors' Contributions}

Si-yi Yu and Zheng-tao Lv contributed equally to this work.

\section{Acknowledgments}

This trial is supported by the programs of the National Natural Science Foundation of China (Grants nos. 81303060 and 81574089), the program of Fok Ying-Tong Education Foundation for Young Teachers in the Higher Education Institutions of China (Grant no. 20155132210002), and the program of People Benefit Project of the Technology Bureau of Chengdu (Grant no. 2015-HM01-00195-SF).

\section{References}

[1] L. French, "Dysmenorrhea," American Family Physician, vol. 71, no. 2, pp. 285-291, 2005.

[2] K. J. Berkley, "Primary dysmenorrhea: an urgent mandate," Pain, vol. 1, pp. 1-8, 2013.

[3] M. Ayan, E. Sogut, U. Tas et al., "Pain levels associated with renal colic and primary dysmenorrhea: a prospective controlled study with objective and subjective outcomes," Archives of Gynecology and Obstetrics, vol. 286, no. 2, pp. 403-409, 2012.

[4] M. Y. Dawood, Dysmenorrhea and Prostaglandins, Springer, New York, NY, USA, 1987.

[5] M. Y. Dawood, "Primary dysmenorrhea: advances in pathogenesis and management," Obstetrics \& Gynecology, vol. 108, no. 2, pp. 428-441, 2006.

[6] S. Iacovides, I. Avidon, and F. C. Baker, "What we know about primary dysmenorrhea today: a critical review," Human Reproduction Update, vol. 21, no. 6, Article ID dmv039, pp. 762778, 2015.

[7] H.-P. Zahradnik, A. Hanjalic-Beck, and K. Groth, "Nonsteroidal anti-inflammatory drugs and hormonal contraceptives for pain relief from dysmenorrhea: a review," Contraception, vol. 81, no. 3, pp. 185-196, 2010.

[8] P. Woo and M. J. Mceneaney, "New strategies to treat primary dysmenorrhea," Clinical Advisor for Nurse Practitioners, 2010.

[9] G. Lefebvre, O. Pinsonneault, V. Antao et al., "Primary dysmenorrhea consensus guideline," Journal of Obstetrics and Gynaecology Canada, vol. 27, no. 12, pp. 1117-1146, 2005.

[10] X.-K. Wu, E. Stener-Victorin, H.-Y. Kuang et al., "Effect of acupuncture and clomiphene in chinese women with polycystic ovary syndrome: a randomized clinical trial," Jama, vol. 317, no. 24, pp. 2502-2514, 2017.

[11] I. Sriprasert, S. Suerungruang, P. Athilarp, A. Matanasarawoot, and S. Teekachunhatean, "Efficacy of acupuncture versus combined oral contraceptive pill in treatment of moderate-to-severe dysmenorrhea: a randomized controlled trial," Evidence-based Complementary and Alternative Medicine, vol. 2015, Article ID 735690, 10 pages, 2015.

[12] G.-X. Shi, Q.-Q. Li, C.-Z. Liu et al., "Effect of acupuncture on Deqitraits and pain intensity in primary dysmenorrhea: analysis of data from a larger randomized controlled trial," $B M C$ Complementary and Alternative Medicine, vol. 14, article 69, 2014.
[13] Y.-X. Ma, X.-N. Ye, C.-Z. Liu et al., "A clinical trial of acupuncture about time-varying treatment and points selection in primary dysmenorrhea," Journal of Ethnopharmacology, vol. 148, no. 2, pp. 498-504, 2013.

[14] U. O. Abaraogu and C. S. Tabansi-Ochuogu, "As acupressure decreases pain, acupuncture may improve some aspects of quality of life for women with primary dysmenorrhea: a systematic review with meta-analysis," JAMS Journal of Acupuncture and Meridian Studies, vol. 8, no. 5, pp. 220-228, 2015.

[15] S. H. Cho and E. W. Hwang, "Acupressure for primary dysmenorrhoea: a systematic review," Bjog An International Journal of Obstetrics \& Gynaecology, vol. 18, no. 1, pp. 49-56, 2010.

[16] Z.-Q. Zhao, "Neural mechanism underlying acupuncture analgesia," Progress in Neurobiology, vol. 85, no. 4, pp. 355-375, 2008.

[17] A. J. Liu, J. H. Li, H. Q. Li et al., "Electroacupuncture for acute ischemic stroke: a meta-analysis of randomized controlled trials," The American Journal of Chinese Medicine, vol. 43, no. 8, pp. 1541-1566, 2015.

[18] D. Mayor, "An exploratory review of the electroacupuncture literature: clinical applications and endorphin mechanisms," Acupuncture in Medicine, vol. 31, no. 4, pp. 409-415, 2013.

[19] H. Z. Zhang, S. L. Shan, W. U. Zhong-Xin, and X. M. Wei, "Effect of electroacupuncture on SP and PGF2 $\alpha$ in serum of dysmenorrhea rats," Journal of Nanjing University of Traditional Chinese Medicine, 2014.

[20] X.-X. Ren, X.-Y. Ding, M.-W. Guo et al., "Effects of instant electroacupuncture at the different acupoints on IP3 in the uterus tissue of dysmenorrhea model rats," Zhongguo Zhen Jiu, vol. 31, no. 1, pp. 60-64, 2011.

[21] L. I. Ling-Yu, P. Yan, and X. F. Jin, "Effect of Acupuncture at Zhibian Point on T Lymphocyte Subsets of Primary Dysmenorrhea Model Rats," Journal of Clinical Acupuncture Moxibustion, 2016.

[22] J. S. Song, Y. Q. Liu, C. Z. Liu et al., "Cumulative analgesic effects of EA stimulation of sanyinjiao (SP 6) in primary dysmenorrhea patients: a multicenter randomized controlled clinical trial," Acupuncture Research, vol. 38, no. 5, 393 pages, 2013 (Chinese).

[23] H. Yang, C.-Z. Liu, H. Yang et al., Systematic Review of Clinical Trials of Acupuncture-Related Therapies for Primary Dysmenorrhea, Centre for Reviews and Dissemination, England, UK, 2008.

[24] Y.-C. Chung, H.-H. Chen, and M.-L. Yeh, "Acupoint stimulation intervention for people with primary dysmenorrhea: Systematic review and meta-analysis of randomized trials," Complementary Therapies in Medicine, vol. 20, no. 5, pp. 353-363, 2012.

[25] M.-N. Chen, L.-W. Chien, and C.-F. Liu, "Acupuncture or acupressure at the sanyinjiao (SP6) acupoint for the treatment of primary dysmenorrhea: A meta-analysis," Evidence-Based Complementary and Alternative Medicine, vol. 2013, Article ID 493038, 8 pages, 2013.

[26] T. Xu, L. Hui, Y. Li-Juan, S. Guo-Min, and W. Tian-Hua, "Effects of moxibustion or acupoint therapy for the treatment of primary dysmenorrhea: A meta-analysis," Alternative Therapies in Health and Medicine, vol. 20, no. 4, pp. 33-42, 2014.

[27] C. A. Smith, M. Armour, X. Zhu, X. Li, Z. Y. Lu, and J. Song, Acupuncture for dysmenorrhoea, John Wiley \& Sons, Ltd, 2016.

[28] Y.-X. Ma, L.-X. Ma, X.-L. Liu et al., "A comparative study on the immediate effects of electroacupuncture at sanyinjiao (SP6), xuanzhong (GB39) and a non-meridian point, on menstrual pain and uterine arterial blood flow, in primary dysmenorrhea patients," Pain Medicine, vol. 11, no. 10, pp. 1564-1575, 2010. 
[29] G.-X. Shi, C.-Z. Liu, J. Zhu, L.-P. Guan, D.-J. Wang, and M.-M. Wu, "Effects of acupuncture at Sanyinjiao (SP6) on prostaglandin levels in primary dysmenorrhea patients," The Clinical Journal of Pain, vol. 27, no. 3, pp. 258-261, 2011.

[30] C. Z. Liu, J. P. Xie, and L. P. Wang, "Immediate analgesia effect of single point acupuncture in primary dysmenorrhea: a randomized controlled trial," Pain Medicine, vol. 12, no. 2, pp. 300-307, 2011.

[31] H. M. Langevin, R. Schnyer, H. Macpherson et al., "Manual and electrical needle stimulation in acupuncture research: pitfalls and challenges of heterogeneity," Journal of Alternative \& Complementary Medicine, vol. 21, no. 3, 113 pages, 2015.

[32] R. Zhang, L. Lao, K. Ren, and B. M. Berman, "Mechanisms of acupuncture-electroacupuncture on persistent pain," Anesthesiology, vol. 120, no. 2, pp. 482-503, 2014.

[33] J. P. T. Higgins, S. G. Thompson, J. J. Deeks, and D. G. Altman, "Measuring inconsistency in meta-analyses," British Medical Journal, vol. 327, no. 7414, pp. 557-560, 2003.

[34] G. Rücker, G. Schwarzer, J. R. Carpenter, and M. Schumacher, "Undue reliance on I2 in assessing heterogeneity may mislead," BMC Medical Research Methodology, vol. 8, article no. 79, 2008.

[35] J. P. T. Higgins and S. G. Thompson, "Quantifying heterogeneity in a meta-analysis," Statistics in Medicine, vol. 21, no. 11, pp. 15391558, 2002.

[36] R. DerSimonian and N. Laird, "Meta-analysis in clinical trials revisited," Contemporary Clinical Trials, vol. 45, pp. 139-145, 2015.

[37] C.-Z. Liu, J.-P. Xie, L.-P. Wang et al., "A randomized controlled trial of single point acupuncture in primary dysmenorrhea," Pain Medicine (United States), vol. 15, no. 6, pp. 910-920, 2014.

[38] G.-X. Shi, C.-Z. Liu, J. Zhu, L.-P. Guan, D.-J. Wang, and M.-M. Wu, "Effects of acupuncture at Sanyinjiao (SP6) on prostaglandin levels in primary dysmenorrhea patients," Clinical Journal of Pain, vol. 27, no. 3, pp. 258-261, 2011.

[39] B. Liu, "Clinical observation of the effectiveness of electroacupuncture for primary dysmenorrhea," Shanxi Journal of Traditional Chinese Medicine, vol. 32, no. 5, p. 36, 2016.

[40] R. Ren and L.-x. Zhuang, "Impact of electroacupuncture on hemorrheology and uterine arteries hemodynamics in treating primary dysmenorrhea," Chinese Archives of Traditional Chinese Medicine, no. 3, pp. 649-651, 2010.

[41] J. B. Song, Y. Q. Liu, and C. Z. Liu, "Cumulative analgesic effect of electroacupuncture at Sanyinjiao (SP6, Xuanzhong (GB39) and non-acupoint for primary dysmenorrhea: a comparative study," Shanghai Journal of Acupuncture and Moxibustion, vol. no. 06, pp. 487-492, 2015.

[42] S. Y. Xin, Y. Q. Liu, and P. Zhang, "Preliminary exploration of relationship between uterine position and meridian point effect specificity in primary dysmenorrhea patients," Shanghai Journal of Acupuncture and Moxibustion, no. 5, pp. 381-383, 2014.

[43] L. X. Zhi, "Randomised controlled study in the analgesic effect of superficial needling plus electrostimulation of Sanyinjiao (SP6) for primary dysmenorrhea," Acupuncture Research, vol. 32, no. 5, pp. 342-346, 2007.

[44] S.A.o.T.C. Medicine, Standards for Diagnosis of Syndromes or Diseases of TCM and Evaluation of the Therapeutic Effect, Nanjing University Press, 1994.

[45] J. S. Han, X. Z. Ding, and S. G. Fan, “Cholecystokinin octapeptide (CCK-8): antagonism to electroacupuncture analgesia and a possible role in electroacupuncture tolerance," PAIN, vol. 27, no. 1, pp. 101-115, 1986.
[46] W. M. Li, K. M. Cui, N. Li et al., "Analgesic effect of electroacupuncture on complete Freund's adjuvant-induced inflammatory pain in mice: A model of antipain treatment by acupuncture in mice," Japanese Journal of Physiology, vol. 55, no. 6, pp. 339-344, 2005.

[47] L.-L. Cheng, M.-X. Ding, J. Wei et al., "Electroacupunctureinduced dynamic processes of gene expression levels of endogenous opioid peptide precursors and opioid receptors in the CNS of goats," Evidence-Based Complementary and Alternative Medicine, vol. 2013, Article ID 257682, 10 pages, 2013.

[48] S. Yu, J. Yang, M. Yang et al., "Application of acupoints and meridians for the treatment of primary dysmenorrhea: A data mining-based literature study," Evidence-Based Complementary and Alternative Medicine, vol. 2015, Article ID 752194, 2015.

[49] Y. P. Yu, "Immediate effect of acupuncture at Sanyinjiao (SP6) and Xuanzhong (GB39) on uterine arterial blood flow in primary dysmenorrhea," Journal of Alternative \& Complementary Medicine, vol. 16, no. 10, pp. 1073-1078, 2010.

[50] M. Yang, X. Chen, L. Bo et al., "Moxibustion for pain relief in patients with primary dysmenorrhea: A randomized controlled trial," PLoS ONE, vol. 12, no. 2, Article ID e0170952, 2017.

[51] Y. U. Chuan, X. U. Shi-Wen, Q. R. Yang, R. Zhang, L. I. GuiXiang, and Y. W. Wang, "Clinical Observations on Premenstrual Acupuncture for Improving Symptoms Accompanying Primary Dysmenorrhea," Shanghai Journal of Acupuncture Moxibustion, 2014.

[52] B. Aicher, H. Peil, B. Peil, and H.-C. Diener, "Pain measurement: Visual Analogue Scale (VAS) and Verbal Rating Scale (VRS) in clinical trials with OTC analgesics in headache," Cephalalgia, vol. 32, no. 3, pp. 185-197, 2012.

[53] U. O. Abaraogu, S. E. Igwe, and C. S. Tabansi-Ochiogu, "Effectiveness of SP6 (Sanyinjiao) acupressure for relief of primary dysmenorrhea symptoms: A systematic review with meta- and sensitivity analyses," Complementary Therapies in Clinical Practice, vol. 25, pp. 92-105, 2016.

[54] C.-Q. Gou, J. Gao, C.-X. Wu et al., "Moxibustion for Primary Dysmenorrhea at Different Interventional Times: A Systematic Review and Meta-Analysis," Evidence-Based Complementary and Alternative Medicine, vol. 2016, Article ID 6706901, 2016.

[55] N. Sut and H. Kahyaoglu-Sut, "Effect of aromatherapy massage on pain in primary dysmenorrhea: A meta-analysis," Complementary Therapies in Clinical Practice, vol. 27, pp. 5-10, 2017.

[56] H. W. Lee, J. H. Jun, K.-J. Kil, B.-S. Ko, C. H. Lee, and M. S. Lee, "Herbal medicine (Danggui Shaoyao San) for treating primary dysmenorrhea: a systematic review and meta-analysis of randomized controlled trials," Maturitas, vol. 85, pp. 19-26, 2016.

[57] M. L. Proctor, C. A. Smith, C. M. Farquhar, and R. W. Stones, "Transcutaneous electrical nerve stimulation and acupuncture for primary dysmenorrhoea," Cochrane Database of Systematic Reviews, vol. 1, no. 1, Article ID CD002123, 2002.

[58] R. Kharaghani, M. M. Rahbari, A. Keramat, M. Mirmohammadkhani, and M. Yallanghach, "The effect of vitamin e on ameliorating primary dysmenorrhea: a systematic review and metaanalysis," Journal of Basic \& Clinical Reproductive Sciences, vol. 3, no. 2, 2015.

[59] C. X. Chen, B. Barrett, and K. L. Kwekkeboom, "Efficacy of oral ginger (zingiber officinale) for dysmenorrhea: a systematic review and meta-analysis," Evidence-Based Complementary and Alternative Medicine, vol. 2016, Article ID 6295737, 10 pages, 2016. 
[60] S. Asmussen, R. Przkora, D. M. Maybauer et al., "Meta-analysis of electroacupuncture in cardiac anesthesia and intensive care," Journal of Intensive Care Medicine, no. 1, Article ID 885066617708558, 2017.

[61] J.-W. Shim, J.-Y. Jung, and S.-S. Kim, "Effects of electroacupuncture for knee osteoarthritis: a systematic review and meta-analysis," Evidence-based Complementary and Alternative Medicine, vol. 2016, Article ID 3485875, 18 pages, 2016.

[62] M. He, X. Li, Y. Liu et al., "Electroacupuncture for tinnitus: a systematic review," PLoS ONE, vol. 11, no. 3, Article ID e0150600, 2016.

[63] K. F. Schulz, I. Chalmers, R. J. Hayes et al., "Dimensions of methodological quality associated with estimates of treatment effects in controlled trials," Jama the Journal of the American Medical Association, vol. 273, no. 5, pp. 408-412, 1995. 


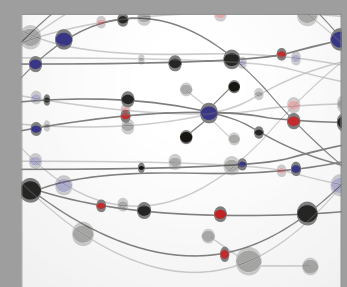

The Scientific World Journal
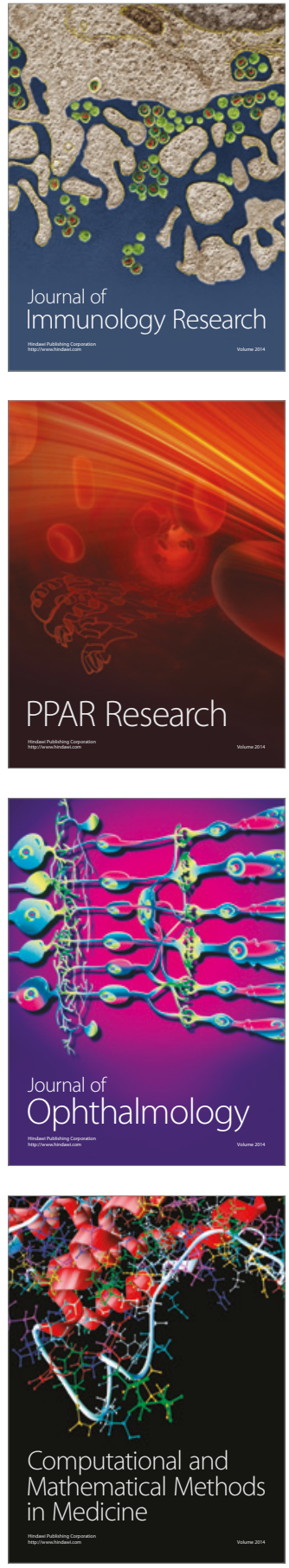

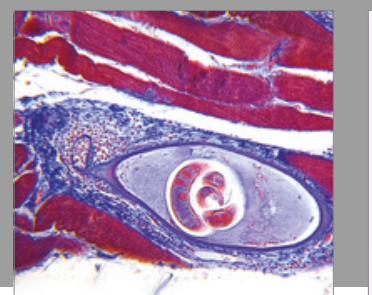

Gastroenterology Research and Practice
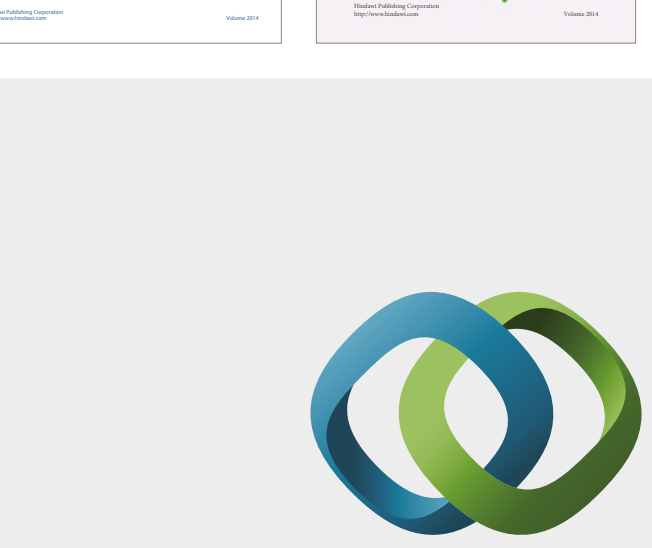

\section{Hindawi}

Submit your manuscripts at

https://www.hindawi.com
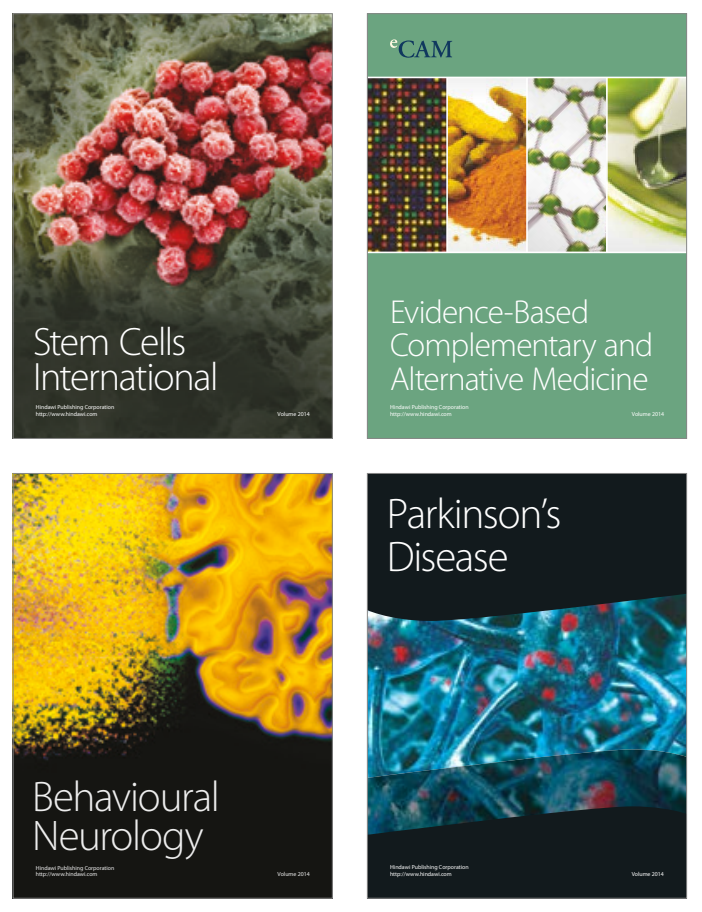
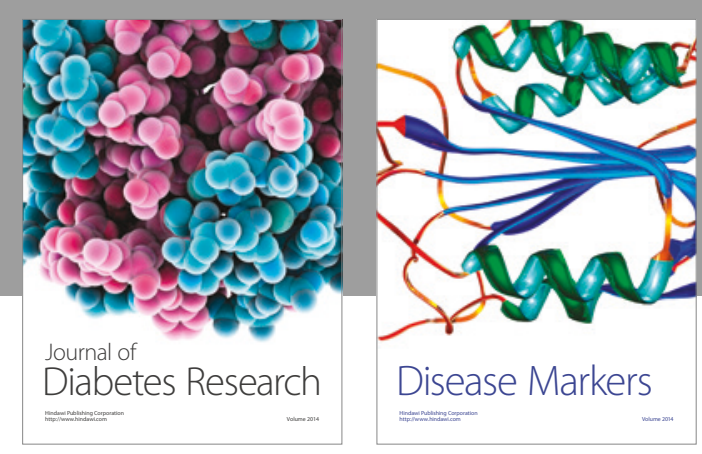

Disease Markers
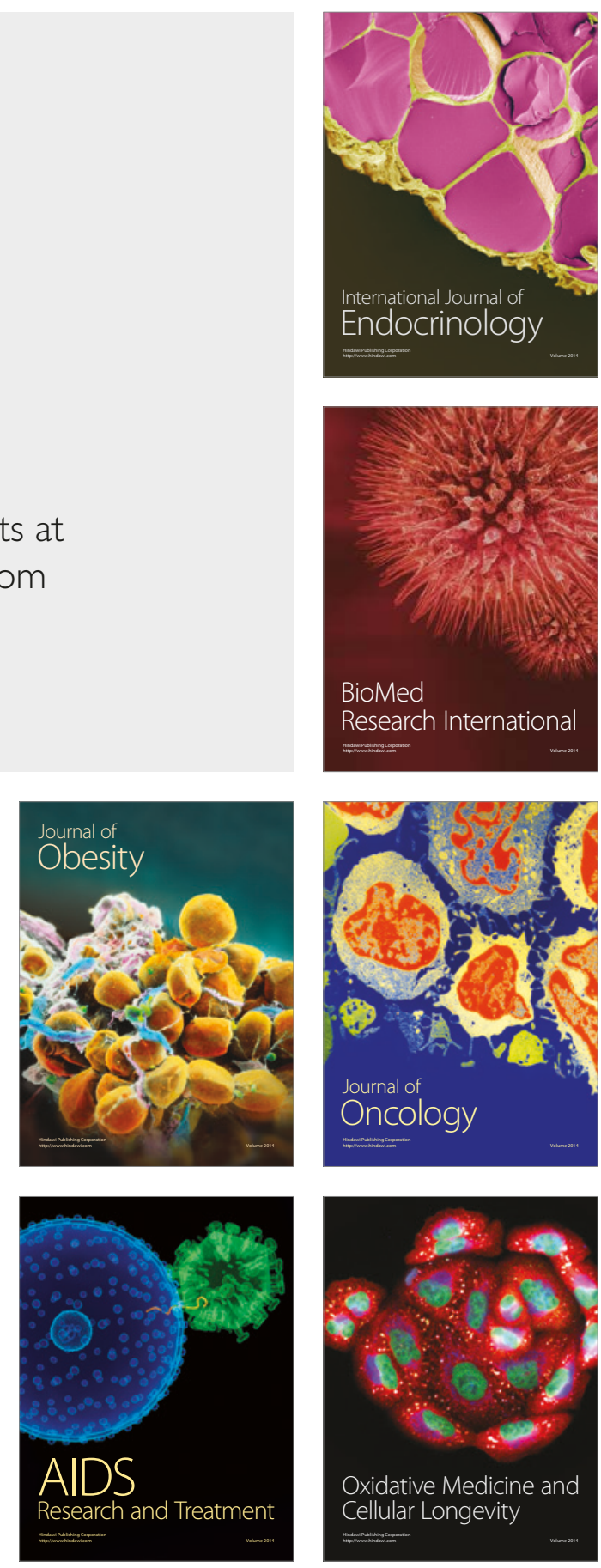